\title{
Combined Effect of Surface Treatment and Surfactant on CNT Nanofluids Preparation
}

\author{
Babita Sharma $^{1}$, S.K. Sharma ${ }^{1}$, Shipra Mital Gupta ${ }^{2}$, Ankita Srivastava ${ }^{1}$, Arinjay Kumar ${ }^{1}$ \\ ${ }^{1}$ USCT, Guru Gobind Singh Indraprastha University, Dwarka, India \\ babita.usct.900210@ipu.ac.in; skschem@ipu.ac.in \\ ${ }^{2}$ USBAS, Guru Gobind Singh Indraprastha University, Dwarka, India \\ shipra@ipu.ac.in
}

\begin{abstract}
CNT nanofluids deliver better rate of heat transfer due to the presence of CNTs which are having high thermal conductivity. Since CNTs are hydrophobic in nature, they entangle themselves and form agglomerates specially in polar solvents. The agglomerates settle down with time and cause reduction in thermo-physical properties and hence lose their potential to deliver better rate of heat transfer. This characteristic of CNT nanofluids requires an efficient preparation method which can provide uniform dispersion of CNTs for a longer duration. This study has been focused on preparation of long term stable CNT nanofluids having effective dispersion of CNTs by the removal of agglomerates which were formed during preparation. To disperse CNTs in water two type of surfactants i.e. sodium dodecylbenzene sulfonate (SDBS) and gum arabic (GA) were used followed by ultrasonication. Based on results, it was analyzed that there is an optimum concentration of both the surfactants which provide maximum dispersion of pristine (p-CNTS) and functionalized (f-CNTS) at particular ultrasonication time. The maximum dispersion concentration of $\mathrm{f}$-CNTs was found to be more than p-CNTs under same conditions. The dispersion of CNTs was more in case when GA was used as surfactant in comparison to SDBS irrespective of ultrasonication time while the thermal conductivity was more for SDBS based CNT nanofluids instead of GA irrespective of type of CNTs. The thermal conductivity of CNT nanofluids was sufficiently high that can be utilized in industries where there is requirement of heat transfer.
\end{abstract}

Keywords: CNT nanofluids, long term stability, fictionalization of CNTs surfactants, ultrasonication, thermal conductivity

\section{Introduction}

Compact and efficient heat transfer systems are the key requirements of production and process industries. Due to enhanced thermal properties, nanofluids have the ability to reduce the size of heat transfer system because they provide the high rate of heat transfer in comparison to conventional fluids [1-5].

Literature reports that due to high thermal conductivity of CNTs, the enhancement in thermal conductivity of CNT based nanofluids is much higher in comparison to other nanoparticles $\left(\mathrm{Al}_{2} \mathrm{O}_{3}, \mathrm{CuO}, \mathrm{TiO}_{2}, \mathrm{SiO}_{2} \mathrm{Cu}, \mathrm{Fe}\right.$, etc. $)$ based nanofluids [1, 6-8]. So, CNT nanofluids appear to be promising as heat transfer fluids for cooling or heating applications in industries. Numerous data is available on the thermal conductivity enhancement of CNT nanofluids. Choi et al. [9] reported $160 \%$ thermal conductivity enhancement by adding 1 vol\% of CNTs in olefin oil. Xie et al. [6] reported 19.6\%, $12.7 \%$ and $7.0 \%$ enhancement in thermal conductivity when functionalized CNTs were dispersed in decene, ethylene glycol and water respectively. Rashmi et al. [10] studied the thermal conductivity of CNT nanofluids and measured up to $250 \%$ enhancement in thermal conductivity.

Though, thermal conductivity of CNT nanofluids is being researched through years, still they have not been industrialized because they are not stable for a longer duration. Under normal conditions CNTs entangled themselves and form agglomerates resulting in a non-homogeneous and unstable suspension when dispersed in conventional fluids. Stability of CNT nanofluids is influenced by nature and quantity of surfactants, functionality of CNT surfaces, size of $\mathrm{CNT}$, ultrasonication frequency and time, etc. [11-14]. If CNTs are treated with strong acids like $\mathrm{HNO}_{3} / \mathrm{H}_{2} \mathrm{SO}_{4} \mathrm{then}$ hydrophilic group like $-\mathrm{OH},-\mathrm{COOH}$, etc. get attached to the CNT surfaces and help in the better dispersion of CNTs in polar solvents in comparison to pristine or purified CNTs (p-CNTs). This happens because the functionalized or treated CNTs have acidic functional group on their surfaces which can dissociate in water and dispersion of CNTs gets enhanced 
by electric repulsion [8, 15-16]. It is also reported that surfactants SDS, SDBS and GA are best surfactants for stabilization of CNTs in polar solvents for a longer time [17]. Sadri et al. [14] prepared CNT nanofluids using GA, SDBS and SDS as surfactants and reported that CNT nanofluids prepared using GA have highest thermal conductivity enhancement in comparison CNT nanofluids prepared using SDS and SDBS. Shanbedi et al. [18] used GA, CTAB and SDS as surfactants for dispersion of CNTs in water. They tried 0.5-1, 1-1 and 2-1 surfactant/MWCNTs ratio to prepare CNT nanofluids and reported that 1-1 surfactant/MWCNTs ratio showed maximum stability irrespective of surfactant.

In the present work, efforts were made to study the combined effect of surface treatment and surfactants for preparing stable CNT nanofluids using SDBS and GA as dispersing agent. Ultrasonication time, type of CNTs and concentration of surfactants were varied to check their effect on dispersion of CNTs. The prepared CNT nanofluids were stored and analyzed for its stability followed by of thermal conductivity.

\section{Experimental Procedure and Apparatus}

Double distilled water was used as basefluid and two type of multi-walled carbon nanotubes (MWCNTs) i.e. pristine CNTs (p-CNTs) and functionalized (-COOH) CNTs (f-CNTs) were used to prepare CNT nanofluids. The CNTs were supplied by Nanostructured \& Amorphous Materials, Inc. USA having specifications - outside diameter 50-80 nm, length 10-20 $\mu \mathrm{m}$, purity $>90 \%$. Sodium dodecyl benzene sulfonte (SDBS) and gum arabic (GA) purchased from HiMedia Laboratories Pvt. Ltd. India, were used as dispersing agent. Ultrasonication was done by using bath ultrasonicaor provided by Golju, Scientific, India.

In order to prepare stable CNT nanofluids, two step method was utilized under dynamic conditions. Initially, stock aqueous surfactant solution was prepared for each surfactant or dispersing agent at $0.685 \mathrm{wt} \%$. Then the next four consequent concentration of surfactant water solution was prepared by diluting the stock solution by adding double distilled water. After that both type of CNTs at constant concentration were added into both type of surfactant water solution while stirring with the help of magnetic stirrer followed by ultrasonication at frequency $40 \pm 3 \mathrm{KHz}$. Ultrasonication time was varied from 1 to 4 hour for each and every sample. To avoid the increase in temperature of CNT nanofluid due to ultrasonication, cooled water was circulated to maintain the bath temperature $\sim 25{ }^{0} \mathrm{C}$. After ultrasonication each sample was centrifuged at $200 \mathrm{rpm}$ for a fixed interval of time to provide dynamic condition. After centrifugation the supernatant was separated from sediment. Then $1 \mathrm{~mL}$ sample was taken out from the supernatant to measure the CNT concentration and rest of the sample were placed for further analysis. The concentration of CNTs was measured using UVspectrophotometer at $800 \mathrm{~nm}$ because at $800 \mathrm{~nm}$ the absorbance due to the presence of surfactant is negligible [19-22]. The thermal conductivity was measured using KD2 Pro thermal property analyzer (Decagon devices, Inc., USA) based on transient hot wire technique at room temperature.

\section{Results and Discussion}

\subsection{Stability of p-CNTs and f-CNTs in Water}

One of the main technical challenges in front of CNT nanofluids is their dispersion in polar solvents like water. To overcome this problem surfactants can be used [17] and mixed in basefluids before adding CNTs into it because surfactants forms non-covalent bond with CNTs and helps to stabilize the CNTs in water [23]. To check the dispersion of p-CNTs and f-CNTs in water two samples of equal concentration of both the CNTs were prepared followed by ultrasonication for same interval of time. It was observed that p-CNTs gradually settled down just after preparation due to hydrophobic nature of pCNTs, Fig. 1a. Acid treated CNTs or f-CNTs showed better dispersion in water in comparison to p-CNTs, Fig. 1b. However, the CNT nanofluid which was prepared using f-CNTs also did not stable for a longer duration. After 24 hours of preparation there was significant settling of f-CNTs, Fig. 1c. So, to prepare long term stable CNT nanofluids and to check the effect of surfactants on the dispersion of p-CNTs and f-CNTs in water, GA and SDBS were used. It was found that the CNT nanofluids prepared using SDBS and p-CNTs, SDBS and f-CNTs, GA and p-CNTs, GA and f-CNTS were stable for more than three months, Fig. 1d, 1e, 1f, and $1 \mathrm{~g}$ respectively. 


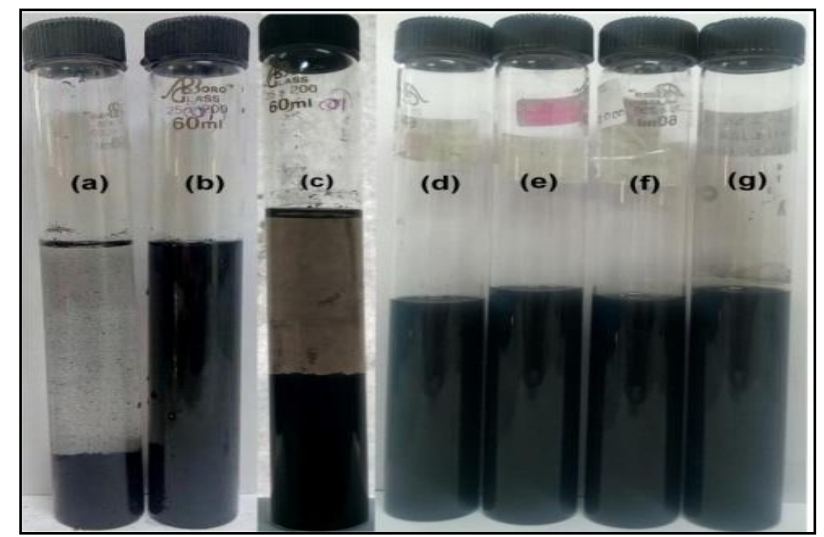

Fig. 1: Stability of (a) p-CNTs in water just after preparation, (b) f-CNTs in water just after preparation, and (c) f-CNTs in water after 24 hours, (d) p-CNTs stabilized using SDBS, (e) f-CNTS stabilized using SDBS, (f) p-CNTs stabilized using GA, and (g) f-CNTs stabilized using GA.

\subsection{Effect of GA and SDBS Concentration on the Dispersion of p-CNTS and f-CNTs in Water}

To study the effect of surfactant concentration on the dispersion of p-CNTs and f-CNTs, the surfactant concentration was varied from 0.137 to $0.685 \mathrm{wt} \%$ keeping p-CNTs and f-CNTs concentration constant $(0.274 \mathrm{wt} \%)$. Results are shown in Fig. 2 to Fig. 5. Fig. 2 and 3 show the effect of SDBS concentration on the dispersion of p-CNTs and f-CNTs in water respectively. It was found that initially the dispersion of both type of CNTs increased with increase in SDBS concentration upto $0.411 \mathrm{wt} \%$ and afterwards dispersion start decreasing. The increase in dispersion of CNTS may be due to the increase in steric hindrance between CNTs surfaces [24]. The decrease in dispersion of CNTs after 0.411 wt\% may be due to the excess amount of SDBS adsorbed on to the surface of CNTs which make the CNT agglomerates bulky and cause settling due to gravitational force [25]. Fig. 4 and 5 show the effect of GA concentration on the dispersion of p-CNTs and f-CNTs in water at different ultrasonication time, respectively. According to fig. 4 and 5, it was observed that with an increase in GA concentration dispersion of p-CNTs and f-CNTs in water increased. This result agrees with the results of Kim et al. [16], as gum arabic adsorbs onto the CNT agglomerates and hence provides repulsive forces between them. The dispersion of both p-CNTs and f-CNTs were maximum at GA concentration $0.548 \mathrm{wt} \%$ and further increase in concentration cause reduction in dispersion.

The maximum dispersion of p-CNTs and f-CNTs in water obtained with different surfactants i.e. SDBS and GA is different. The maximum dispersion of p-CNTs and f-CNTs was obtained when GA concentration is 0.548 wt $\%$ but for SDBS the maximum dispersion of p-CNTs and f-CNTs was obtained at $0.411 \mathrm{wt} \%$ of SDBS. This concentration difference may be due to difference in surfactant behavior or their structure. It also may be due to the difference in mechanism of adsorption of both of the surfactant on CNTs surfaces [24]. The concentration of p-CNTs and f-CNTs is greater when GA used as surfactant in comparison to CNT nanofluids prepared using SDBS. According to Hao et al. [26] each surfactant has its own best concentration and capability to stabilize the nanofluids for a longer duration.

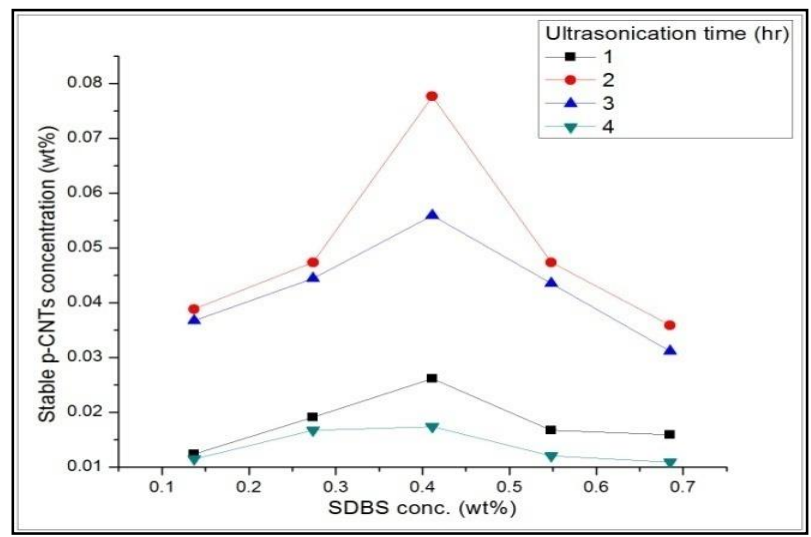

Fig. 2: Effect of SDBS concentration on the dispersion of p-CNTs in water at different ultrasonication time. 


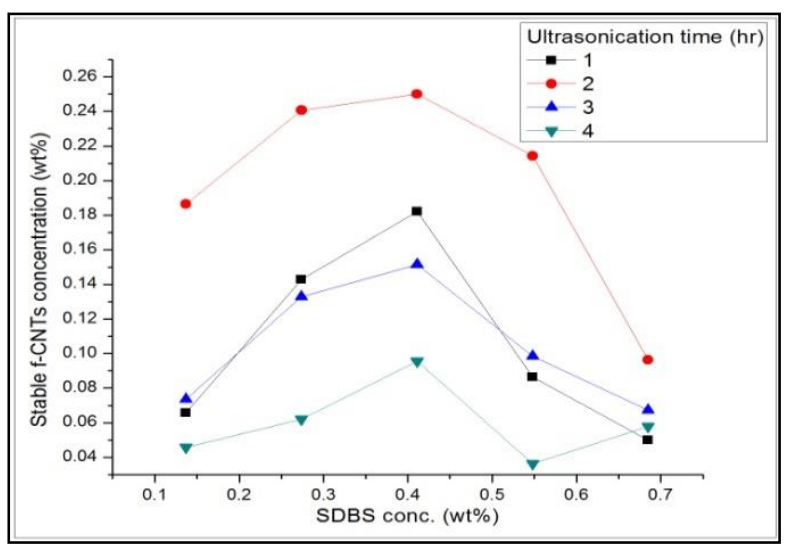

Fig. 3: Effect of SDBS concentration on the dispersion of f-CNTs in water at different ultrasonication time.

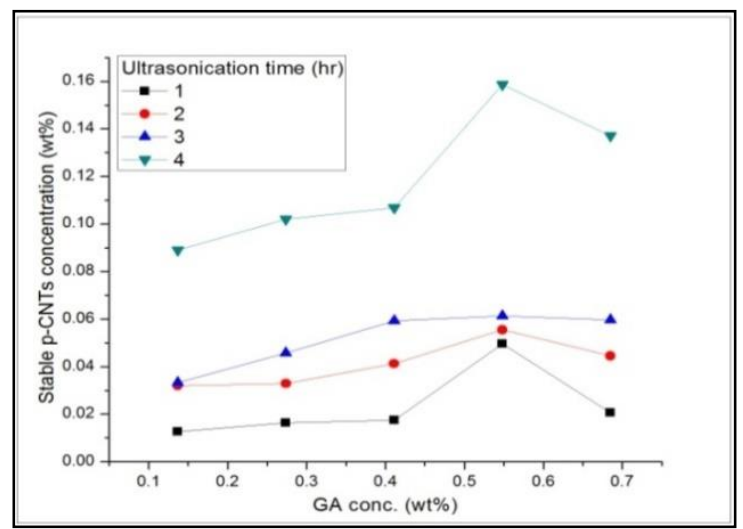

Fig. 4: Effect of GA concentration on the dispersion of p-CNTs in water at different ultrasonication time.

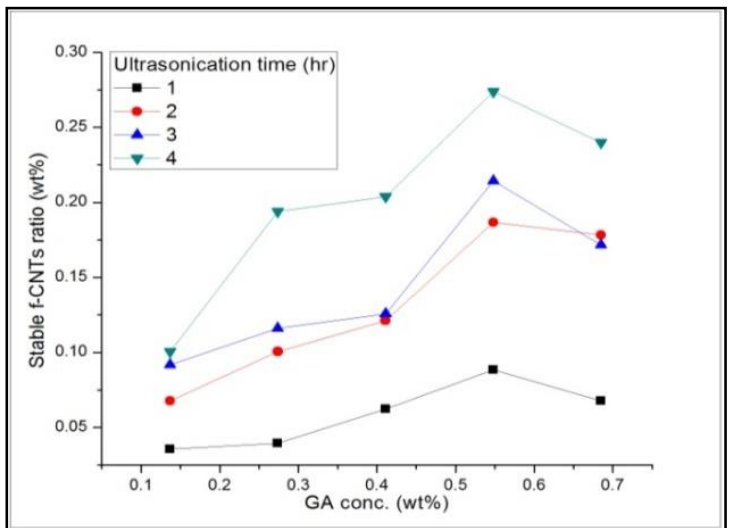

Fig. 5: Effect of GA concentration on the dispersion of f-CNTs in water at different ultrasonication time.

\subsection{Effect of Ultrasonication Time on the Dispersion of $p-C N T S$ and $f-C N T s$ in Water}

Since, CNTs form agglomerates and entangle themselves and it is very difficult to disperse them in basefluids without the use of any physical treatment like ultrasonication [27-308-31] or mixing at high shear [31-332-34]. Both the processes are governed by the transfer of shear stress to break the agglomerates of CNTs [34] and have a significant effect on the dispersion of CNTs in basefluids. So, to study the effect of ultrasonication time on the dispersion of p-CNTs and f-CNTs in water it was varied from 1 to 4 hour for SDBS and GA concentration 0.137 to $0.685 \mathrm{wt} \%$. Fig. 6 and 7 show the effect of ultrasonication time on the dispersion of p-CNTs and f-CNTs in water at constant SDBS concentration. It is observed that with increase in ultrasonication time dispersion of p-CNTs and f-CNTs increased and at 2 hour ultrasonication, dispersion is maximum. Effect of ultrasonication time on CNT nanofluids is attributed to the breakup of CNT agglomerates into 
smaller clusters of CNTs. Also, increased ultrasonication time leads to an increase in the dispersion of CNTs in basefluids [14]. Further increase in ultrasonication time leads to the reduction in the dispersion of p-CNTs and f-CNTs. It may be due to the fact that, providing excess ultrasonication to CNT nanofluids leads to a decrease in aspect ratio of CNTs. Also, prolonged sonication effect the structure of surfactants or the micro-SDBS particles might be formed of SDBS which was already adsorbed on to surface of CNTs and due to Vander Waals forces the micro particles of SDBS accumulate themselves and form packages which resulted in sedimentation of CNTs or SDBS [25] Fig. 8 and 9 show the effect of ultrasonication time on the dispersion of p-CNTs and f-CNTs in water at constant GA concentration. It is observed that with increase in ultrasonication time dispersion of p-CNTs and f-CNTs in water increased. It is reported in literature that, 4 hour ultrasonication is sufficient for the CNT nanofluids prepared using GA. Further ultrasonication leads to the reduction in CNTs dispersion due to damage of CNT structure which includes bending, bucking or dislocations in carbon structure [10].

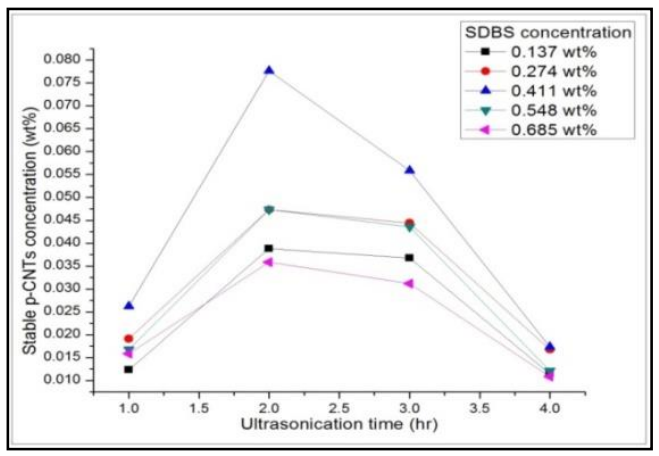

Fig. 6: Effect of ultrasonication time on the dispersion of f-CNTs in water at different SDBS concentration.

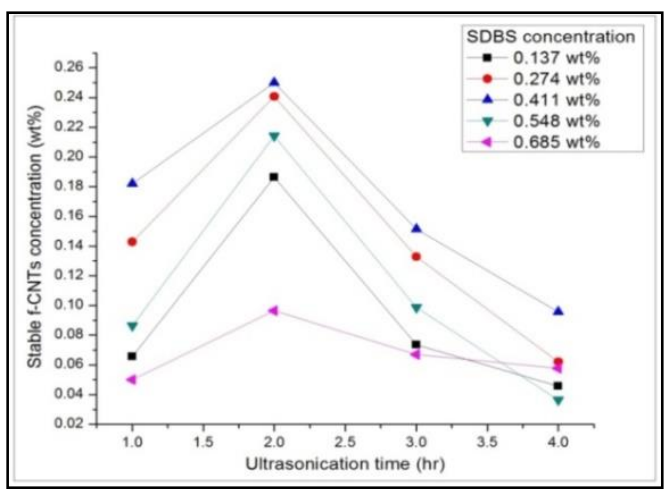

Fig. 7: Effect of ultrasonication time on the dispersion of f-CNTs in water at different SDBS concentration.

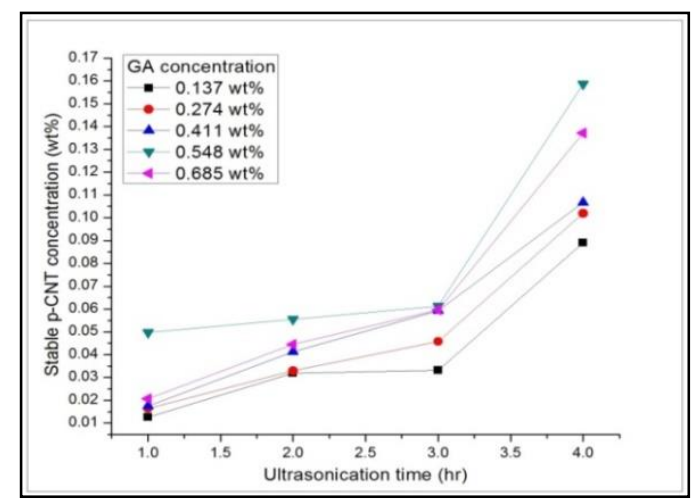

Fig. 8: Effect of ultrasonication time on the dispersion of p-CNTs in water at different GA concentration. 


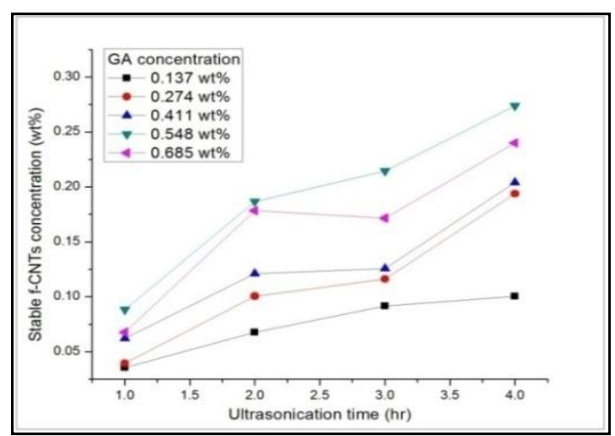

Fig. 9: Effect of ultrasonication time on the dispersion of f-CNTs in water at different GA concentration.

\subsection{Effect of Type of CNTs on the Dispersion of p-CNTs and f-CNTs in Water}

To study the dispersion of CNTs in water, CNT nanofluids were prepared using two type of CNTs i.e. p-CNTs and fCNTs of same specifications. Results are shown in Fig. 10 and 11 for dispersion of both type of CNT nanofluids prepared using SDBS and GA, respectively and results were compared in terms of CNT dispersion. It is observed that dispersion of $\mathrm{f}$-CNTs is more than p-CNTs at constant surfactant concentration and at particular ultrasonication time. This may be due to the fact that on the surface of $\mathrm{f}-\mathrm{CNTs}$, $-\mathrm{COOH}$ group is attached due to acid treatment of p-CNTS and which causes conversion of hydrophobic to hydrophillic. It is reported in literature that during acid treatment carboxyl group attached to the CNTs surfaces leads to breaking of CNTs into shorter with less twisted shreds, hence results in better dispersion of fCNTs in basefluids. There are no van der Waals forces between f-CNTs because of ionization of acidic surface group on $\mathrm{f}$ CNTs which increases the electrostatic repulsion between them and make them more dispersible in basefluids in comparison to p-CNTs $[6,13,15,21]$.

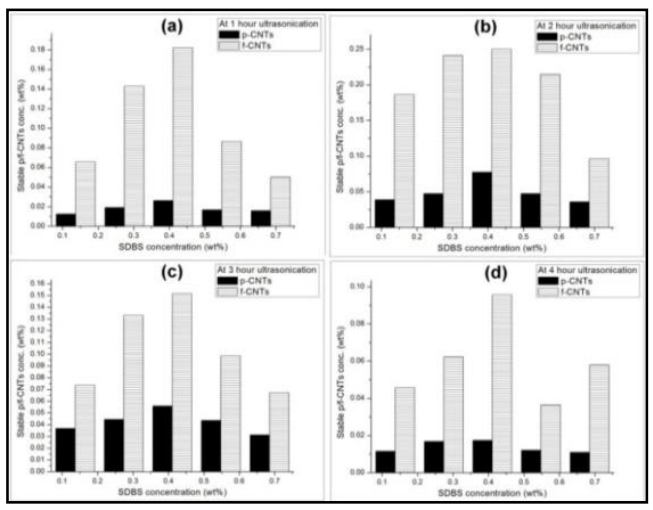

Fig. 10: Comparison of p-CNTs and f-CNTs dispersion in water with change in SDBS concentration at ultrasonication (a) $1 \mathrm{hr}$, (b) $2 \mathrm{hr}$, (c) $3 \mathrm{hr}$ and (d) $4 \mathrm{hr}$.

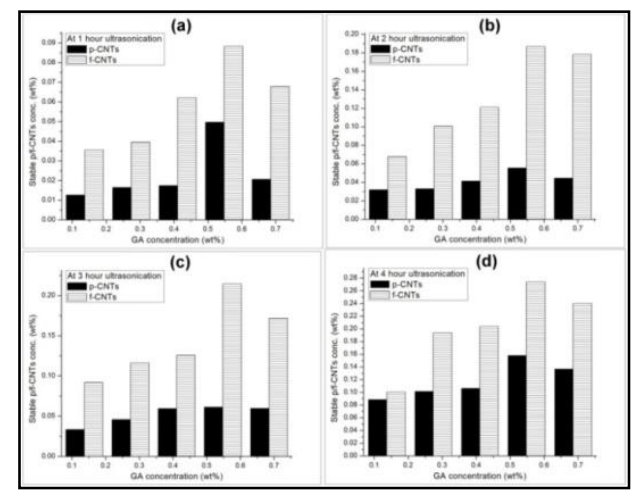

Fig. 11: Comparison of p-CNTs and f-CNTs dispersion in water with change in GA concentration at ultrasonication (a)1 hr, (b)2 hr, (c) $3 \mathrm{hr}$ and (d) $4 \mathrm{hr}$. 


\subsection{Thermal Conductivity of CNT Nanofluids}

The thermal conductivity of CNT nanofluids is reported in Fig. 12 in terms of thermal conductivity enhancement. The enhancement in thermal conductivity is calculated as follow:

$$
\% \text { Thermal conductivity enhancement }=\frac{\mathbf{k}_{\mathrm{CNT} \text { nanofluid }}-\mathbf{k}_{\text {water }}}{\mathbf{k}_{\text {water }}} * 100
$$

The thermal conductivity of CNT nanofluids was measured only for those samples which gave maximum dispersion of p-CNTs and f-CNTs with SDBS and GA, Fig. 12. The optimum condition at which there was maximum dispersion of pCNTs and f-CNTs was $0.411 \mathrm{wt} \%$ conc. and $2 \mathrm{hr}$ ultrasonication time, $0.548 \mathrm{wt} \%$ conc. and $4 \mathrm{hr}$ ultrasonication time for SDBS and GA based CNT nanofluids respectively. Figure 12 shows that there is a large thermal conductivity enhancement of CNT nanofluids in comparison to water irrespective of type of CNTs and surfactant. From the Fig. it is analyzed that there is a little difference in thermal conductivity enhancement when CNT nanofluids prepared using p-CNTs and f-CNTs irrespective of surfactant. But, when comparison is done on the basis of dispersion of CNTs then it is more for $\mathrm{f}$-CNTs compared to p-CNTs. It means there is a non-linear relation between thermal conductivity and concentration of CNT nanofluids $[35,36]$. Also, thermal conductivity of CNT nanofluids prepared using SDBS is more compared to GA based CNT nanofluids irrespective of type of CNTs while the dispersion of CNTs in more for GA based CNT nanofluids. This indicates that thermal conductivity of CNT nanofluids not only depends on the concentration of CNTs but also dependent on the type of surfactant and its concentration, ultrasonication time, etc. [36].

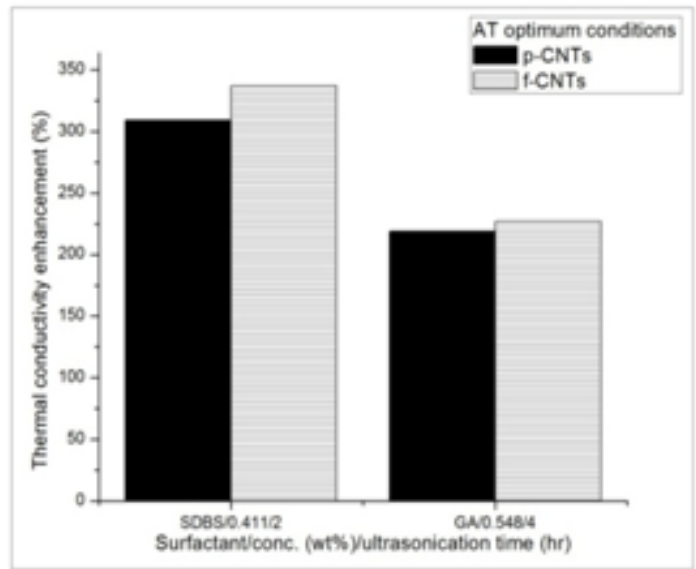

Fig. 12: Thermal conductivity of CNT nanofluids at optimum conditions.

\section{Conclusions}

In this study the combined effect of surface treatment and surfactants were investigated to check the dispersion of CNTs in water. The dispersion of p-CNTs and f-CNTs is dependent on the type of surfactant and concentration of surfactant. Also, ultrasonication time has a significant effect on the dispersion of CNTs. Different surfactants have their own best concentration to disperse CNTs in water. There is more dispersion of f-CNTs in water compared to p-CNTs. Enhancement in thermal conductivity found to be more for SDBS based CNT nanofluids than GA based CNT nanofluids irrespective of type of CNTs. In addition to CNT concentration thermal conductivity of CNT nanofluids also depends on type and concentration of surfactant, ultrasonication time, stability, etc.. The prepared CNT nanofluids were found to be stable for more than three months.

\section{Acknowledgement}

This study is financial supported by GGSIP University, Dwarka India under FRGS project. 


\section{References}

[1] Y. Hwang, H. S. Park, J. K. Lee and W. H. Jung, "Thermal conductivity and lubrication characteristics of nanofluids," Curr. Appl. Phys. vol. 6, pp. e67-e71, 2006.

[2] H. T. Zhu, C. Y. Zhang, Y. M. Tang and J. X. Wang, "Novel Synthesis and Thermal Conductivity of CuO Nanofluid," J. Phys. Chem. C., vol. 111, pp. 1646-1650, 2007.

[3] I. Tavman, A. Turgut, M. Chirtoc, H.P. Schuchmann and S. Tavman, "Experimental investigation of viscosity and thermal conductivity of suspensions containing nano-sized ceramic particles," Archives Mater. Sci. Eng., vol. 34, no. 2, pp. 99-104, 2008.

[4] W. Duangthongsuk and S. Wongwises, "Measurement of temperature-dependent thermal conductivity and viscosity of $\mathrm{TiO}_{2}$-water nanofluids," Exp. Therm. Fluid Sci., vol. 33, pp. 706-714, 2009.

[5] S. Harish, K. Ishikawa, E. Einarsson, S. Aikawa, S. Chiashi, J. Shiomi and S. Maruyama, "Enhanced thermal conductivity of ethylene glycol with single-walled carbon nano-tube inclusions," Int. J. Heat Mass Tran., vol. 55, pp. 3885-3890, 2012.

[6] H. Xie, H. Lee, W. Youn and M. Choi, "Nanofluids containing multi-walled carbon nano-tubes and their enhanced thermal conductivities," J. Appl. Phys., vol. 94, no.8, pp. 4967-4971, 2003.

[7] A. Nasiri, M. Shariaty-Niasar, A. M. Rashidi and R. Khodafarin, "Effect of CNT structures on thermal conductivity and stability of nanofluid," International Journal of Heat and Mass Transfer, vol. 55, pp. 1529-1535, 2012.

[8] L. Chen, H. Xie, Y. Li and W. Yu, "Nanofluids containing carbon nanotubes treated by mechanochemical reaction," Thermochimica Acta, vol. 477, pp. 21-24, 2008.

[9] S. U. S. Choi, Z.G. Zhang, W. Yu, F. E. Lockwood and E. A. Grulke, "Anomalous thermal conductivity enhancement in nanotube suspensions," Appl. Phys. Lett., vol. 79, no. 14, pp. 2252-2254, 2001.

[10] W. Rashmi, A. F. Ismail, I. Sopyan, A. T. Jameel, F. Yusof, M. Khalid and N. M. Mubarak, "Stability and thermal conductivity enhancement of carbon nanotube nanofluid using gum arabic," J. Exp. Nanosci., vol. 6, pp. 567-579, 2011.

[11] B. S. K. Sharma, S. M. Gupta, "Preparation and evaluation of stable nanofluids for heat transfer application: A review," Experimental Thermal and Fluid Science, vol. 79, pp. 202-212, 2016.

[12] A. Nasiri, M. S. Niasar, A. Rashidi, A. Amrollahi, R. Khodafarin, "Effect of dispersion method on thermal conductivity and stability of nanofluid," Exp. Therm. Fluid Sci., vol. 35, pp. 717-723, 2011.

[13] M. N. A. W. N Yazid, N. A. C. Sidik, R. Mamat, G. Najafi, "A review of the impact of preparation on stability of carbon nanotube nanofluids," International Communications in Heat and Mass Transfer, vol. 78, pp. 253-263, 2016.

[14] R. Sadri, G. Ahmadi, H. Togun, M. Dahari, S. N. Kazi, E. Sadeghinezhad, N. Zubir, "An experimental study on thermal conductivity and viscosity of nanofluids containing carbon nanotubes," Nanoscale Res. Lett., vol. 9, no. 151, 2014.

[15] K. Esumi, M. Ishigamai,, A. Nakajima, K. Sawada, H. Honda, "Chemical treatment of carbon nanotubes," Carbon, vol. 34, no. 2, pp. 279-281, 1996.

[16] M. T. Kim, H. S. Park, D. Hui, K. Y. Rhee, "Carbon Nanotube Modification Using Gum Arabic and Its Effect on the Dispersion and Tensile Properties of Carbon Nanotubes/Epoxy Nanocomposites," Journal of Nanoscience and Nanotechnology, vol. 11, pp. 7369-7373, 2011.

[17] Y. Ding, H. Alias, D. Wen, R. A. Williams, "Heat transfer of aqueous suspensions of carbon nanotubes (CNT nanofluids)," Int. J. Heat Mass Tran., vol. 49, pp. 240-250, 2006.

[18] M. Shanbedi, S. Z. Heris, A. Maskooki, "Experimental investigation of stability and thermophysical properties of carbon nanotubes suspension in the presence of different surfactants," J. Therm. Anal. Calorim., vol. 120, pp. 11931201, 2015.

[19] T. P. Teng, Y. B. Fang, Y. C. Hsu, L. Lin, "Evaluating stability of aqueous multiwalled carbon nanotube nanofluids by using different stabilizers," J. Nanomater., 2014.

[20] Y. Kun, Y. Z. Li, J. Q. Feng, Y. R.. Liang, J. Wei, L. D. Hui, "Sonication-assisted dispersion of carbon nanotubes in aqueous solutions of the anionic surfactant SDBS: The role of sonication energy," Chin. Sci. Bull., vol. 58, no. 17, pp. 2082-2090, 2013.

[21] L. Ju, W. Zhang, X. Wang, J. Hu, Y. Zhang, "Aggregation kinetics of SDBSdispersed carbon nanotubes in different aqueous suspensions," Colloids Surf., A, vol. 409, pp. 159-166, 2012. 
[22] O. Matarredona, H. Rhoads, Z. Li, J. H. Harwell, L. Balzano, D. E. Resasco, "Dispersion of single-walled carbon nanotubes in aqueous solutions of the anionic surfactant NaDDBS," J. Phys. Chem. B, vol. 107, pp. 13357-13367, 2003.

[23] W. Yu and H. Xie, "A Review on Nanofluids: Preparation, Stability Mechanisms and Applications," J. Nanomater., vol. 2012, pp. 1-17, 2012.

[24] L. Vaisman, H. D. Wagner, G. Marom, "The role of surfactants in dispersion of carbon nanotubes," Advances in Colloid and Interface Science, vol. 128, no. 130, pp. 37-46, 2006.

[25] Q. Y. Tang, I. Shafiq, Y. C. Chan, N. B. Wong, R. Cheung, "Study of the Dispersion and Electrical Properties of Carbon Nanotubes Treated by Surfactants in Dimethylacetamide," J. Nanosci. Nanotechnol., vol. 10, pp. 4967-4974, 2010.

[26] S. J. Hao, Y. Z. Zhang, W. F. Jiang, Z. L. Pang, "Stability of aqueous suspension containing carbon nanotubes," Dongbei Daxue Xuebao/J. Northeast. Univ., vol. 28, no. 10, pp. 1438-1441, 2007.

[27] Y. Y. Huang and E. M. Terentjev, "Dispersion and rheology of carbon nanotubes in polymers," Int. J. Mater. Form., vol. 1, pp. 63-74, 2008.

[28] Y. Y. Huang, T. P. J. Knowles, E. M. Terentjev, "Strength of nanotubes, filaments, and nanowires from sonicationinduced scission," Adv. Mater., vol 21, pp. 3945-3948, 2009.

[29] Y. Yamamoto, Y. Miyauchi, J. Motoyanagi, T. Fukushima, T. Aida, M. Kato and S. Maruyama, "Improved bath sonication method for dispersion of individual single-walled carbon nanotubes using new triphenylene-based surfactant," Jpn. J. Appl. Phys., vol. 47, pp. 2000-2004, 2008.

[30] R. Ramasubramaniama, J. Chen, "Homogeneous carbon nanotube/polymer com-posites for electrical applications," Appl. Phys. Lett., vol. 83, pp. 2928-2930, 2003.

[31] R. Andrews, D. Jacques, M. Minot and T. Rantell, "Fabrication of carbon multi-wallnanotube/polymer composites by shear mixing," Macromol. Mater. Eng., vol. 287, pp. 395-403, 2002.

[32] J. H. Park, P. S. Alegaonkar, S. Y. Jeon, J. B. Yoo, "Carbon nanotube composite: Dispersion routes and field emission parameters," Compos. Sci. Technol., vol. 28, pp. 753-759, 2008.

[33] M. H. G.Wichmann, J. Sumeth, B. Fiedler, F. H. Gojny, K. Schulte, "Multi-wall carbon nanotube/epoxy composites produced with a masterbatch process," Mech. Comp. Mater., vol. 42, pp. 395-406, 2006.

[34] Y. Yan Huang, E. M. Terentjev, "Dispersion of Carbon Nanotubes: Mixing, Sonication, Stabilization, and Composite Properties," Polymers, vol. 4, pp. 275-295, 2012.

[35] D. Wen, Y. Ding, "Experimental investigation into convective heat transfer of nanofluids at the entrance region under laminar flow conditions," Int J Heat Mass Transf, vol. 47, pp. 5181-5188, 2004.

[36] R. Walvekar, I. A. Faris, M. Khalid, "Thermal Conductivity of Carbon Nanotube Nanofluid-Experimental and Theoretical Study," Heat Transfer-Asian Research, vol 41, no. 2, 2012. 